\title{
Linear and non linear study of a possible mechanism for the generation of stellar radio bursts: THE SYNCHROTRON MASER INST ABILITY
}

\author{
P.Louarn,D.Le Queau and A.koux
}

\section{CNET/CRPE ,38-10 Rue du General Leclerc \\ 92131 ISSY LES MOULINEAUX}

\begin{abstract}
Abune: The emission mechanism studied here, the synchrotron maser instability is based on a gyroresonance of highly energetic electrons. Compared to the cyclotron maser, this mechanism has the great advantage to be a direct amplifination process even if the ratio $(1)_{p}(1) c_{c}$ is not very small; a situation relevant to stellar flares. The high efficiency of the process can be confirm by the non linear study of its saturation.
\end{abstract}

\section{1) INTRODUCTION:}

The cyclotron maser instability is now widely aknowledged as being the appropriate mechanism for explaining planetary radio emissions. This instability, linked to the relativistic gyroresonance of energetic electrons $(10 \mathrm{KeV})$ and fed by positive $\partial f / \partial v_{\perp}$ slopes in the electronic distribution function, as also been invoked for explaining certain solar and stellar radio bursts (Melrose and Dulk, 1982). This interpretation, however, suffers from serious difficulties (Shama and Vlahos, 1984):

(i) In a low $\omega_{p} /()_{c}$ p plasma $\left({ }^{\left.()_{p} / /\right)_{c}<0}<3\right)$, the most amplified waves are emitied on the $X$ mode, at frequencies closed to the gyrofrequency. These waves are reabsorbed by the hot background plasma when, during their propagation they cross the layer where their frequency is twice the local gyrofrequency. (ii) In a higher $\omega_{\mathrm{p}} / \omega_{\mathrm{c}}$ plasmat, the $\mathrm{Z}$ mode is the most amplified one. As this mode can not escape out of the plasmat, a conversion mechanism is needud for explaining the emission. This conversion is however expected to greally reduce the effiency of the whole process.

The mechanism proposed here: the synchrotron maser, could constitute a solution to these problems. Indeed, this instability, based un a gyroresonance of highly energetic electrons (up to $1 \mathrm{MeV}$ ), predominantly amplifies the Xmode, even if the ratio $\omega_{\mathrm{p}} /\left(\mathrm{c}_{\mathrm{c}}\right.$ is not very low. This result is presented in section II, devoted to the linear study of the instability. An estination of the efficiency of the process is made in section III and finally, a discussion and the conclusion are proposed (section IV). 


\section{II ) LINEAR STUDY OF THE} INSTABILITY:

- Basic Remarksi The formal expression of the growth rate of the instability is the same as the cyclotron maser one. It can be written:

$$
\begin{array}{r}
\gamma=A\left(\omega_{p}, w_{c}, \omega\right) \sum_{m} \int d^{3} \vec{p} J_{m}^{2}\left(r \frac{k_{1} v_{L}}{\omega_{c}}\right) . \\
\cdot B\left(\frac{\partial g}{\partial v_{1}}, k, \omega\right) \cdot \delta\left(\omega-k_{1} v_{11}-m \frac{\omega_{c}}{\Gamma}\right)
\end{array}
$$

(For an exact expression of the different coefficients, see Wu, 1985)

The growth rate of the instability is the result of a summation on the successive resonant pole contributions. The main differences between the cyclotron maser and the synchrotron maser instability come from this summation. In the cyclotron maser case, the clectrons have low energies $(\Gamma \approx 1)$; the resonance is then only fullfilled near the gyroharmonics and the growth rate is calculaled by using an unic resonant pole. Conversely, in the synchrotron maser case, a wave with a given frequency $\omega$ can resonate with many different electrons having energies defined by: $\Gamma_{\mathrm{m}}=\mathrm{m} . \omega_{\mathrm{c} / \omega}$. The contributions of several resonamt poles must then be taken into account in the calculation of the wave growth rate (Figure). See Louarn et al ,1986 for at more complete discussion.

-Silculation of the Growth Rate: The results concerning these calculations are described in a short article: Lowarn et al 1987. The main conclusions are the following:

(i) The value of $\omega_{p} / \omega_{c}$ for which the $Z$ moxk becomes predominant increases with the energy of the electrons. This value can reach 3 or 4 for an energy of $1 \mathrm{MeV}$ (Figure 2).

(ii) The frequency bandwidth of the emission is large compared to the one obtained with the cyclotron maser (10-20\% instead of $1 \%$ ). In certain condition, pseudo harmonics can be obtained (Louam et al 1986).

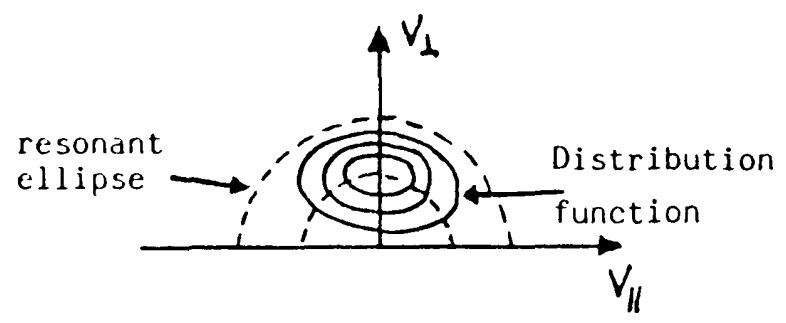

Cyclotron maser: one resonant pole.

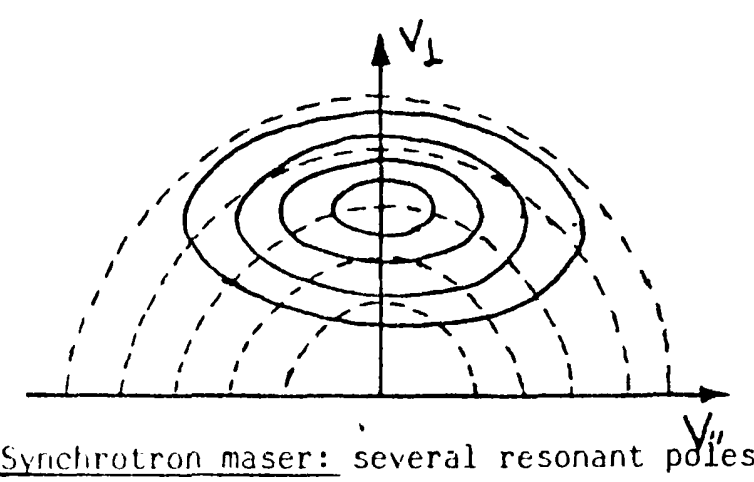

rigure 1.

The very inter esting consequence of this linear study is that the synchrotron mase: directly amplifies the $\mathrm{X}$ mode above the second or the third gyroharmonic in conditions where $\omega_{\mathrm{p}} / \omega_{\mathrm{c}}$ is not very small. Then, during its propagation, the radiation is not severely reabsorted by the background plasma.

An estimation of the efficiency of the process, obtained by the study of the non-linear saturation of the instability, is now presented.

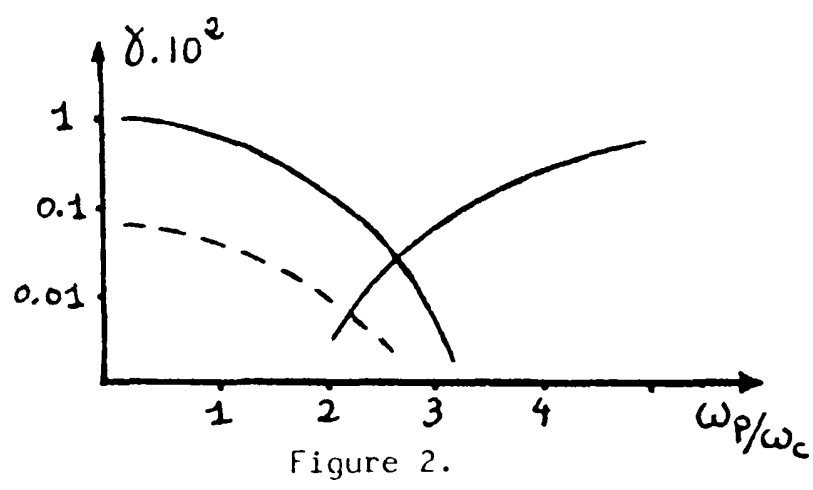

Maximum growth rates of the 3 modes $x$, $O$ and $z$, as function of $\omega p / \omega c$ (from Louarn et al., 1987). 
III) NON LINEAR EYOLUTION OF THE

\section{INSTABILITY:}

This study is made by using the quasi-linear diffusion theory. We only consider the most amplified waves ( $X$ mode in perpendicular propagation) and then the equations describing the evolution of the instability write:

$\frac{\partial F}{\partial t}=\pi q^{e} \frac{1}{P_{1}} \frac{\partial}{\partial P_{\perp}}\left[\int \frac{d^{3} k}{(2 \pi)^{3}} \sum_{m} P_{1} \delta\left(\omega-m \frac{w_{c}}{r}\right) E_{k, m} E_{k, m} \frac{\partial F}{\partial P_{\perp}}\right]$ with $\left.E_{k, m}=\frac{1}{\sqrt{2}}\left[\xi_{k}^{+} e^{i \varphi_{J_{m+1}}}\left(r \frac{t_{1} V_{d}}{\omega_{c}}\right)+\varepsilon_{k}^{-} e^{-i \varphi_{J}}\right]_{m-1}\right]$ $\frac{\partial W_{e}}{\partial t} \cdot m=\pi^{2} q^{2} \int \frac{d^{3} k}{\left(2 \pi^{3}\right.} \int d P_{1} d P_{\|} \sum_{m} \frac{p_{1}^{2}}{r_{m_{D}}} \delta\left(w-m \frac{w_{c}}{r}\right)$. . $\varepsilon_{k m} \varepsilon^{*} k_{i m} \frac{\partial F}{\partial P_{\perp}}$

These equations can be simplified by supposing that during its saturation, the spectral width of the emission does not change. The diffusion operateur $D\left(p_{\perp}\right)$ is then easily calculated; it can be approximated by a simple function: $\mathrm{D}\left(\mathrm{p}_{\perp}\right)=K \mathrm{p}_{\perp} \alpha$. The system of equations becomes:

$$
\begin{gathered}
\frac{\partial E}{\partial t}=-k_{1}\left[\int d u_{\perp} 1 / \Gamma_{3} v_{\perp}^{\alpha}\left(\alpha v_{\perp}^{2}+\alpha+1\right) F\left(v_{\perp}\right)\right] \xi(t) \\
\frac{\partial F}{\partial t}=k_{2} \frac{1}{v_{1}} \frac{\partial}{\partial v_{1}}\left[u_{\perp}^{\alpha} \xi(t) \frac{\partial F}{\partial u_{1}}\right] \\
u_{1}=\frac{P_{1} / m_{0} c}{}
\end{gathered}
$$

For $\alpha=-1 / 2$, a value that relatively well fits $D\left(p_{\perp}\right)$, a solution of this system is:

$$
\begin{aligned}
F(\tau, x) & =A e^{-\left(\frac{x}{\xi}\right)^{7 / 4}}\left(1-\Delta e^{-\left(\frac{x}{E \xi}\right)^{7 / 4}}\right) \\
\text { with } \xi & =\left(\xi_{0}^{7 / 4}+\zeta\right)^{4 / 7} ; \varepsilon=\frac{\varepsilon}{\xi_{0}}\left(\xi_{0}^{7 / 4}+\xi+\frac{\xi}{\xi_{0} / 4}\right) \\
\text { and } \xi & =\int_{0}^{\zeta} \frac{(7 / 4)^{2}}{4 k_{2}} \zeta\left(\tau^{\prime}\right) d \zeta^{\prime} \\
x & =U_{\perp}^{2} \text { and } \quad \zeta=\omega_{c} t
\end{aligned}
$$

Evolution of the electromagnetic field

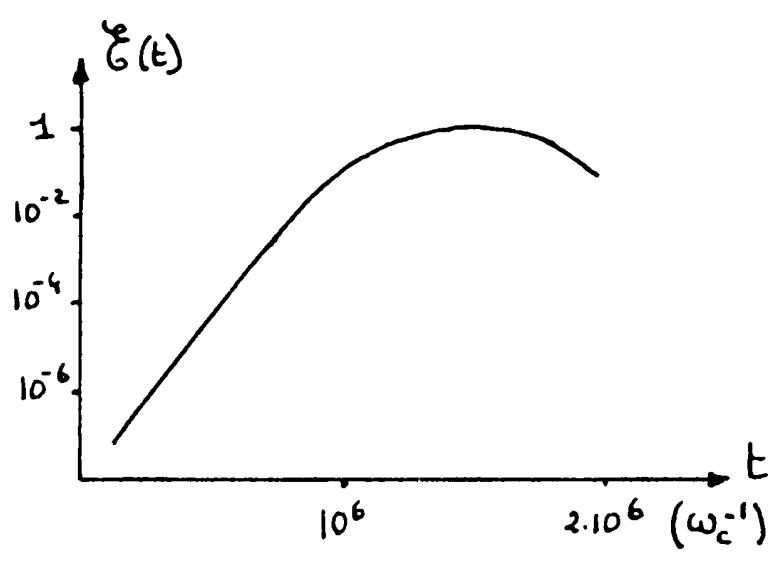

Evolution of the distribution

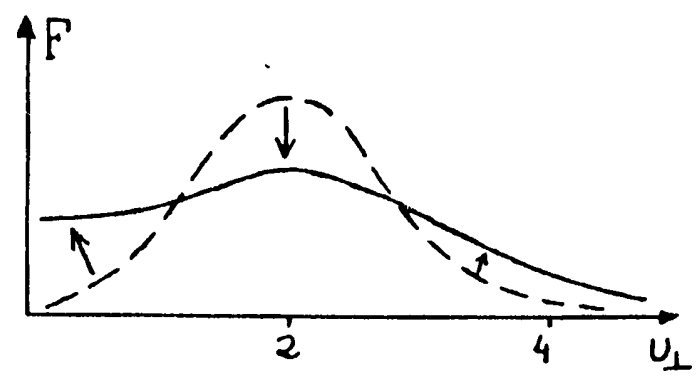

Figure 3. Evolution of the system for $\alpha=-1 / 2$

The time evolution of both the electromagnetic field and the electronic distribution function are shown on figure 3.

A typical exponentiation time of the instability is around: $10^{-2} \cdot n_{c} / n_{h} \cdot \omega_{c}{ }^{-1}$ (where $n_{h / n_{c}}$ is the ratio between the hot and the cold electron iensity). The efficiency of the process can reach $1 \%$, a value similar to those obtained with the cyclotron maser. The saturation can be extremely rapid: starting with a small amount of electromagnetic energy compared to the kinetic one $\left(10^{-8}\right)$, the saturation is reached

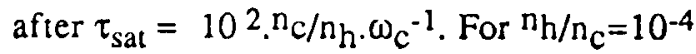
and $\omega_{c}=10^{9}$, this corresponds to $10^{-3}$ second. 


\section{IVLISCUSSIONAND CONSILSION}

From both the lincar and the non linear siucly of the inceatility a rough extrmation of the brighthese kemperature of the emasion cart tre obtaned. Supposing that $1 \%$ of the energy of $1 \mathrm{M} \cdot \mathrm{V}$ electoms contained in a volume $\mathrm{L}^{3}$ (uhere l is $\tau_{\text {wol }}($ ) is converted into a radiation taving a sectral width of $10 \%$ and an angular appertire of 20 , the brightenes temperature of the exper ted enmsion wosdd be approximately 11).4 nh, $n_{0} k^{\prime}$

The mechanism itselt is conseguently quite efficient, howerer, the existence of 1 MeV electroms prescanting an inversion of population (d)/d, $: 0(1)$ is sitll quentionable If such dheributione are unlikely proxiuced during solar thates, the stuation could, housever, be completely differch in case of wher objects flates stars or hinary systems, where catrencels strong acceleration mechanisms

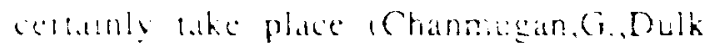
(i. A. $193-2$.

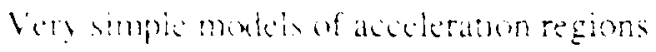
catti then tx. magned llagure 4 in which the cumbinatom of an acceletation dice to a

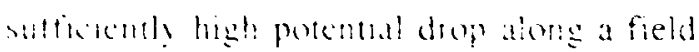

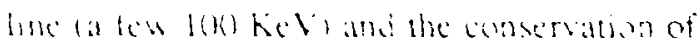

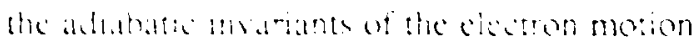

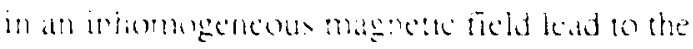
formastur of unstable datribution tametrons. In the presence of sueth distributions a bright cmisson, with a relatively barge spectiom and an high polarization. will be certaingy produced by the syotwotron maser instabilts.

\section{Mode! of the acceleration region :}

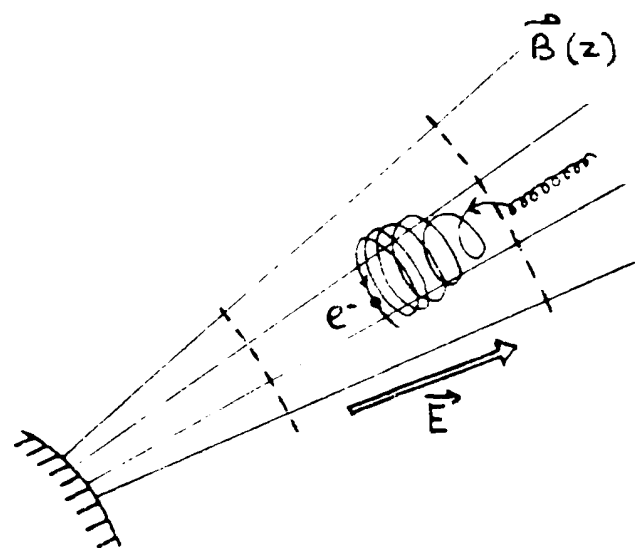

Distribution obtalned inside the arcelelation region:

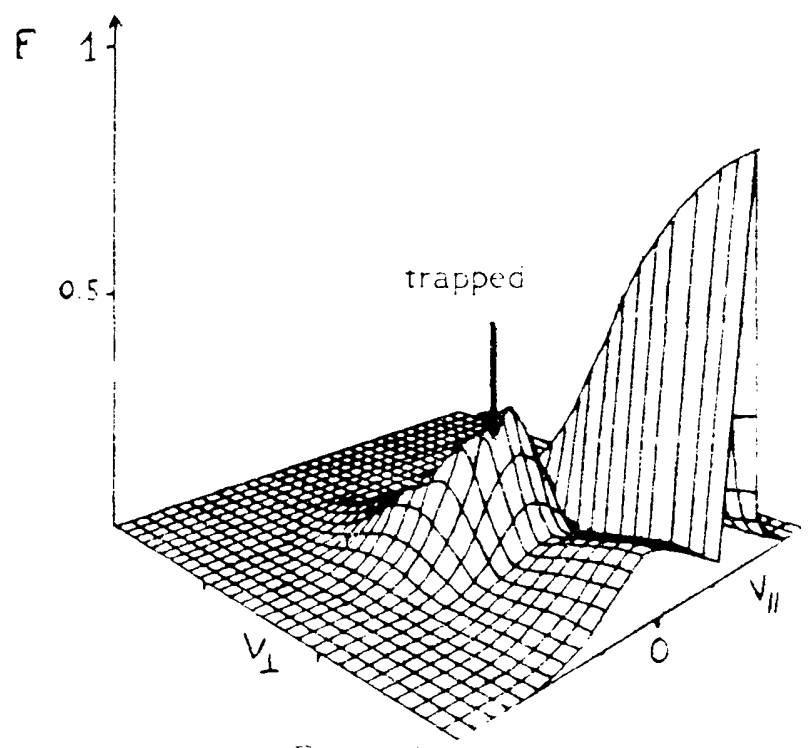

Figure 4.

\section{Reforence:}

Chanmugan.G.Dulk.G.A.:1982,4strophy J.57.285

Louam et al: 1986. Astron.Astrophys., 165.211

Louith t't al: 1987 Solar Phys.,111,201

Melrow D.B.,Dulk G.A.:1982,Astrophy J.,259,844

Sharma.R.R., Wlahos.L.: 1984. Astrophy J.,280,405

Wu. C.S.1985Space Sciences Rex. 41,215 